\title{
A NOVEL PROGNOSTIC INDEX IN PATIENTS WITH RESECTABLE ESOPHAGEAL SQUAMOUS CELL CARCINOMA: FIBRINOGEN/PREALBUMIN RATIO
}

\author{
JI-Feng Feng ${ }^{1,2}$, LIANG WANG ${ }^{1}$, You-Hua Jiang ${ }^{1,2}$ and XUN YANG ${ }^{1,2}$ \\ ${ }^{1}$ Department of Thoracic Oncological Surgery, Institute of Cancer Research and Basic Medical Sciences, \\ Cancer Hospital, University of the Chinese Academy of Sciences (Zhejiang Cancer Hospital); ${ }^{2}$ Key Laboratory \\ Diagnosis and Treatment Technology in Thoracic Oncology, Zhejiang Cancer Research Institute, Zhejiang Cancer \\ Hospital, Hangzhou, China
}

\begin{abstract}
Background: Fibrinogen (Fib) to albumin (ALB) fibrinogen-to-albumin ratio as a prognostic index for esophageal cancer has been confirmed. A novel prognostic index was initially proposed with fibrinogen to prealbumin ratio (FPR) in patients with resectable esophageal squamous cell carcinoma (ESCC). Objective: The objective of the study was to study the prognostic role of the novel prognostic index (FPR) in patients with resectable ESCC without any neoadjuvant treatment. Methods: In this retrospective study, a total of 372 resectable ESCC patients without any neoadjuvant treatment were included. The best cutoff values were selected by the receiver operating characteristic curves. Two Cox regression analyses with forward stepwise (one for categorical variables and the other for continuous variables) were used to evaluate the overall survival (OS) and cancer-specific survival (CSS). Results: The best cutoff point was 0.014 for FPR. Patients with lower levels of FPR $(\leq 0.014)$ had better CSS $(50.7 \%$ vs. $18.0 \%, p<0.001)$ and OS $(48.0 \%$ vs. $17.6 \%, p<0.001)$ than patients with higher levels of FPR (>0.014). Multivariate Cox analyses (categorical and continuous) demonstrated that FPR was an independent prognostic factor in CSS (categorical: hazard ratio [HR]: 2.014, 95\% confidence interval [Cl]: 1.504-2.697, p < 0.001; continuous per 0.01: HR: 1.438, 95\% $\mathrm{Cl}: 1.154-1.793, \mathrm{p}=0.001$ ) and OS (categorical: HR: 1.964, 95\% Cl: 1.475-2.617, $\mathrm{p}<0.001$; continuous per 0.01: HR: 1.429 , $95 \% \mathrm{Cl}: 1.146-1.781, p=0.002$ ). Conclusions: Our study indicated that FPR served as an independent prognostic factor in patients with resectable ESCC. (REV INVEST CLIN. 2020;72(1):46-54)
\end{abstract}

Key words: Esophageal squamous cell carcinoma. Fibrinogen. Albumin. Prealbumin. Cancer-specific survival. Overall survival. Prognosis.

\section{INTRODUCTION}

As one of the most prevalent cancers, esophageal cancer (EC) is the $6^{\text {th }}$ leading cause of cancer mortality worldwide ${ }^{1,2}$. Esophageal squamous cell carcinoma
(ESCC) is the most common pathological type of EC in China ${ }^{3}$. Although radical surgical resection is the standard and effective treatment, the prognosis in patients with EC remains poor ${ }^{4,5}$. To predict the prognosis of EC; therefore, it is increasingly urgent and
*Corresponding author:

Ji-Feng Feng

E-mail: Fengjf@zjcc.org.cn
Received for publication: 16-07-2019

Approved for publication: 17-09-2019

DOI: $10.24875 /$ RIC.19003184

0034-8376 / (c) 2019 Revista de Investigación Clínica. Published by Permanyer. This is an open access article under the CC BY-NC-ND license (http://creativecommons.org/licenses/by-nc-nd/4.0/). 
important to explore more useful and effective prognostic biomarkers.

It has been reported that fibrinogen (Fib), a major protein in the blood clotting process, is associated with cancer ${ }^{6}$. Although recent studies have reported that plasma Fib was related to cancer progression and prognosis in various cancers ${ }^{7-9}$, the role of plasma Fib in EC remains controversial ${ }^{10-12}$. Recently, two metaanalyses revealed that plasma Fib was a prognostic indicator in patients with $\mathrm{EC}^{13,14}$. As a nutritional factor, albumin (ALB) reflected the nutritional status in a variety of cancers, including patients with $\mathrm{EC}^{15,16}$. A new prognostic index in recent years was proposed with fibrinogen-to-albumin ratio (FAR) for patients with $\mathrm{ESCC}^{17}$.

Prealbumin (PALB) is another important factor for nutritional status, which is more sensitive to malnutrition than ALB $^{18,19}$. According to the above theoretical basis; therefore, we hypothesized that PALB could substitute for ALB to combine with Fib to construct a new index: fibrinogen to prealbumin ratio (FPR). A novel prognostic index with FPR was initially proposed to predict the overall survival (OS) and cancer-specific survival (CSS) in patients with resectable ESCC patients.

\section{METHODS}

\section{Patients}

Between January 2006 and December 2010, a retrospective study, including 372 patients with resectable ESCC was conducted in our department. Inclusion criteria were: (1) patients with ESCC in TNM Stage I-III confirmed by histopathology; (2) patients with curative surgical treatment performed without any neoadjuvant treatment; (3) patients without any form of inflammatory diseases; and (4) preoperative Fib, ALB, PALB, neutrophil count, and lymphocyte count obtained 1 week before surgery. Informed consent regarding the collection of specimens was signed by each patient before surgery. The Zhejiang Cancer Hospital Ethics Committee approved the current study in June 2018 (IRB2018-130). All patients in this study participated in accordance with the principles embodied in the Declaration of Helsinki.

\section{Treatment and follow-up}

All of the patients underwent radical esophagectomy with lymphadenectomy. The standard esophagectomy, including the Ivor-Lewis and McKeown procedures, was determined by the tumor location ${ }^{20,21}$. The two-field was the main method of lymphadenectomy used $^{22}$. Patients with any neoadjuvant treatment were excluded from the study. Post-operative adjuvant therapy was not mandatory because adjuvant therapy was still controversial during that period (2006-2010). Cisplatin and 5-fluorouracil were the most frequent drugs for adjuvant chemotherapy. A total dose of 50-60 Gy for postoperative radiotherapy was delivered in 25-30 fractions (2.0 Gy/fraction). The follow-up was performed at regular intervals in all patients (every 3 months for the first 2 years, every 6 months for the next 3 years, then annually). The last follow-up date was June 2014.

\section{Data collection and measurement}

The clinical characteristics, including gender, age, vessel invasion, tumor location, differentiation, tumor length, TNM stage, PALB, ALB, Fib, neutrophil count, and lymphocyte count, were collected. The TNM stage was in accordance with the $7^{\text {th }}$ AJCC/UICC TNM staging system ${ }^{23}$. The levels of Fib, PALB, ALB, neutrophil count, and lymphocyte count were obtained within 1 week before surgery. Neutrophil and lymphocyte counts were performed by automated blood cell counter (Sysmex XE-2100, Kobe, Japan). The level of Fib was determined by automatic coagulation analyzer (Sysmex CA-7000, Kobe, Japan). Levels of ALB and PALB were measured by latex-enhanced homogeneous immunoassay (Hitachi 917; Skill, Munich, Germany).

\section{Statistical analysis}

The best cutoff points for NLR, Fib, PALB, ALB, FAR, and FPR were selected by receiver operating characteristic (ROC) curves according to the CSS status (alive or death). The Chi-squared test was used to analyze the categorical variables of characteristics grouped by FPR. CSS and OS were evaluated with Cox regression analyses (forward stepwise regression). Two models for Cox analyses (one for categorical variables and the other for continuous variables) were utilized to determine hazard ratio (HR) with 95\% 
Figure 1 . ROC curves analyses regarding the best cutoff values and AUC areas. The best cutoff values for Fib, ALB, PALB, FAR, FPR, and NLR were $3.95 \mathrm{~g} / \mathrm{L}, 42.0 \mathrm{~g} / \mathrm{L}, 235.0 \mathrm{mg} / \mathrm{L}, 0.1,0.014$, and 3.76, respectively. The AUC areas were $0.739,0.711$, $0.712,0.653,0.608$, and 0.640 for FPR, FAR, Fib, NLR, ALB, and PALB, respectively.

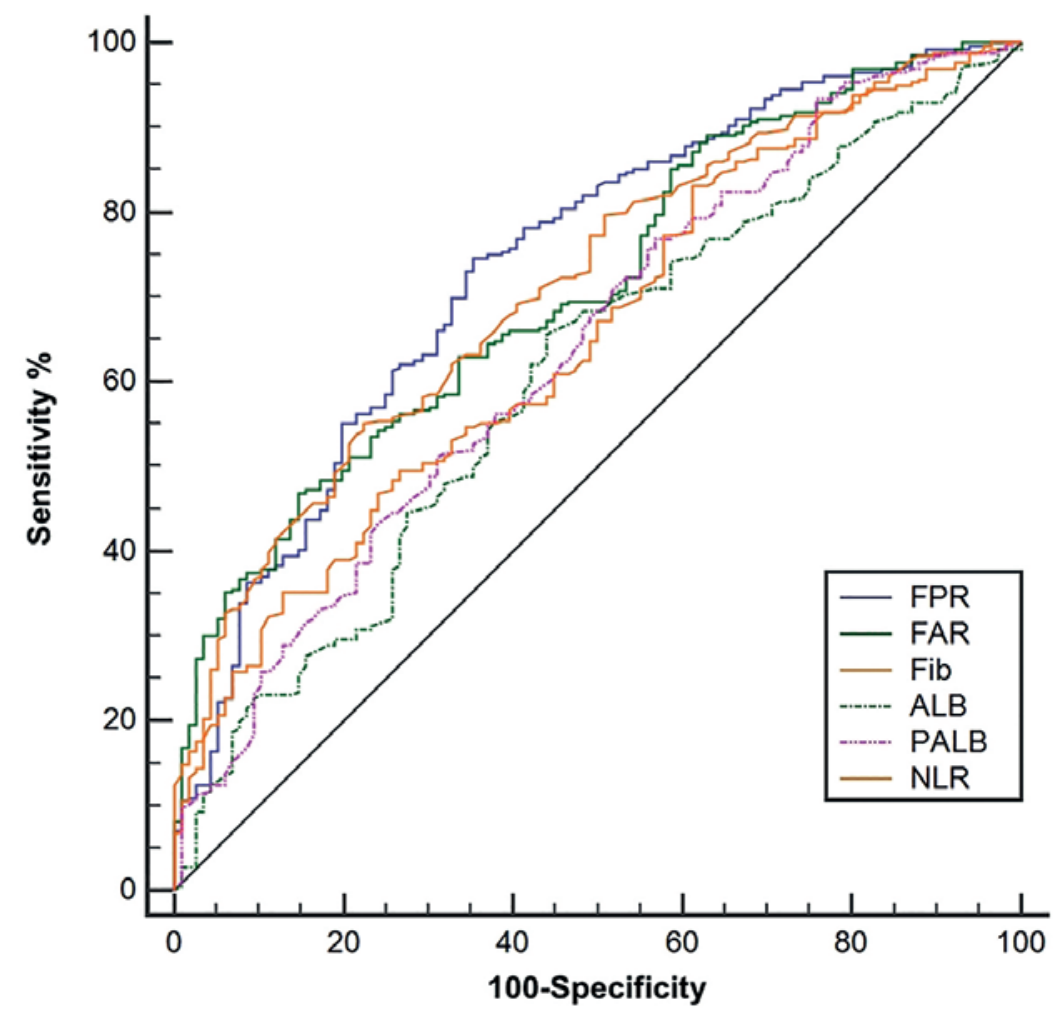

confidence interval $(\mathrm{Cl})$ for CSS and OS $24-26$. The areas under the curve (AUC) for NLR, Fib, FAR, FPR, ALB, and PALB were calculated and compared. A nomogram model was performed with R 3.6 .0 software. MedCalc 15.2 (MedCalc Software bvba, Ostend, Belgium) and SPSS 20.0 (SPSS Inc., Chicago, IL, USA) were performed to analyze the statistical analyses.

\section{RESULTS}

\section{Patient characteristics}

The clinicopathologic characteristics are shown in table 1 . According to clinical criteria, a total of 115 patients $(30.9 \%)$ received post-operative adjuvant radiotherapy and/or chemotherapy. The best cutoff values for Fib, PALB, ALB, FAR, FPR, and NLR were 3.95 $\mathrm{g} / \mathrm{L}, 235.0 \mathrm{mg} / \mathrm{L}, 42.0 \mathrm{~g} / \mathrm{L}, 0.1,0.014$, and 3.76, respectively (Fig. 1). According to the cutoff level, patients were then divided into two groups (FPR $\leq$ 0.014 and FPR > 0.014) (Table 1 ). In our study, the FPR was statistically significantly associated with
TNM stage, ALB, PALB, NLR, Fib, and FAR. Pearson's correlation analyses revealed that FPR was positively correlated with Fib $(r=0.673, p<0.001)$, FAR $(r=$ $0.622, p<0.001)$, and NLR $(r=0.206, p<0.001)$, but was negatively correlated with ALB ( $r=-0.175$, $p=0.001)$ and PALB ( $r=-0.713, p<0.001)$ (Fig. 2).

\section{CSS and OS analysis}

At the last follow-up time, 261 (70.2\%) of the 372 patients had died. The median follow-up time and survival time were 73 months and 32 months, respectively. Patients with lower levels of FPR $(\leq 0.014)$ had better CSS (50.7\% vs. $18.0 \%, \mathrm{p}<0.001$ ) and OS ( $48.0 \%$ vs. $17.6 \%, p<0.001$ ) (Fig. 3 A and B). In subgroup analyses based on the TNM stage (TNM I, TNM II, and TNM III), we found that FPR was also significantly related to CSS (Fig. 3C-E) and OS (Fig. $3 \mathrm{~F}-\mathrm{H}$ ). Using categorization in multiple groups (instead of two) would help to define a biological gradient; therefore, we divided FPR into three groups $(\leq 0.01,0.01-0.02$, and $>0.02)$. The results also 
Table 1. Comparison of the clinical characteristics according to fibrinogen/pre-albumin ratio in esophageal squamous cell carcinoma patients

\begin{tabular}{|c|c|c|c|c|}
\hline & \multirow[t]{2}{*}{ Cases $(n, \%)$} & \multicolumn{2}{|c|}{ FPR } & \multirow[t]{2}{*}{ p-value } \\
\hline & & Low $(n, \%)$ & High (n, \%) & \\
\hline \multicolumn{5}{|l|}{ Age (years) } \\
\hline$\leq 60$ & $210(56.5)$ & $78(52.0)$ & $132(59.5)$ & 0.155 \\
\hline$>60$ & $162(43.5)$ & $72(48.0)$ & $90(40.5)$ & \\
\hline Gender & & & & 0.711 \\
\hline Female & $45(12.1)$ & $17(11.3)$ & $28(12.6)$ & \\
\hline Male & $327(87.9)$ & $133(88.7)$ & $194(87.4)$ & \\
\hline \multicolumn{5}{|l|}{ Tumor length $(\mathrm{cm})$} \\
\hline$\leq 3.0$ & $106(28.5)$ & $48(32.0)$ & $58(26.1)$ & 0.218 \\
\hline$>3.0$ & $266(71.5)$ & $102(68.0)$ & $164(73.9)$ & \\
\hline Tumor location & & & & 0.901 \\
\hline Upper & $25(6.7)$ & $9(6.0)$ & $16(7.2)$ & \\
\hline Middle & $172(46.2)$ & $70(46.7)$ & $102(45.9)$ & \\
\hline Lower & $175(47.1)$ & $71(47.3)$ & $104(46.9)$ & \\
\hline Vessel invasion & & & & 0.136 \\
\hline Negative & $312(83.9)$ & $131(87.3)$ & $181(81.5)$ & \\
\hline Positive & $60(16.1)$ & $19(12.7)$ & $41(18.5)$ & \\
\hline Perineural invasion & & & & 0.076 \\
\hline Negative & $293(78.8)$ & $125(83.3)$ & $168(75.7)$ & \\
\hline Positive & $79(21.2)$ & $25(16.7)$ & $54(24.3)$ & \\
\hline Differentiation & & & & 0.064 \\
\hline Well & $52(14.0)$ & $22(14.7)$ & $30(13.5)$ & \\
\hline Moderate & $246(66.1)$ & $107(71.3)$ & $139(62.6)$ & \\
\hline Poor & $74(19.9)$ & $21(14.0)$ & $53(23.9)$ & \\
\hline TNM stage & & & & 0.001 \\
\hline I & $94(25.3)$ & $47(31.3)$ & $47(21.2)$ & \\
\hline$\|$ & $119(32.0)$ & $56(37.4)$ & $63(28.4)$ & \\
\hline III & $159(42.7)$ & $47(31.3)$ & $112(50.4)$ & \\
\hline $\mathrm{ALB}(\mathrm{g} / \mathrm{L})$ & $40.94 \pm 5.46$ & $41.95 \pm 4.69$ & $40.26 \pm 5.84$ & 0.002 \\
\hline$\leq 42.0$ & $219(58.9)$ & $79(52.7)$ & $140(63.1)$ & 0.046 \\
\hline$>42.0$ & $153(41.1)$ & $71(47.3)$ & $82(36.9)$ & \\
\hline PALB (mg/L) & $252.9 \pm 62.0$ & $301.3 \pm 53.0$ & $222.3 \pm 43.5$ & $<0.001$ \\
\hline$\leq 235.0$ & $175(47.0)$ & $19(12.7)$ & $156(70.3)$ & $<0.001$ \\
\hline$>235.0$ & $197(53.0)$ & $131(87.3)$ & $66(29.7)$ & \\
\hline NLR & $3.48 \pm 3.28$ & $2.79 \pm 1.85$ & $3.95 \pm 3.90$ & $<0.001$ \\
\hline$\leq 3.76$ & $266(71.5)$ & $124(82.7)$ & $142(64.0)$ & $<0.001$ \\
\hline$>3.76$ & $106(28.5)$ & $26(17.3)$ & $80(36.0)$ & \\
\hline $\mathrm{Fib}(\mathrm{g} / \mathrm{L})$ & $3.80 \pm 0.85$ & $3.31 \pm 0.55$ & $4.13 \pm 0.85$ & $<0.001$ \\
\hline$\leq 3.95$ & $232(62.4)$ & $125(83.3)$ & $107(48.2)$ & $<0.001$ \\
\hline$>3.95$ & $140(37.6)$ & $25(16.7)$ & $115(51.8)$ & \\
\hline FAR & $0.095 \pm 0.028$ & $0.080 \pm 0.015$ & $0.105 \pm 0.030$ & $<0.001$ \\
\hline$\leq 0.1$ & $239(64.2)$ & $132(88.0)$ & $107(48.2)$ & $<0.001$ \\
\hline$>0.1$ & $133(35.8)$ & $18(12.0)$ & $115(51.8)$ & \\
\hline
\end{tabular}

ESCC: esophageal squamous cell carcinoma, ALB: albumin, PALB: prealbumin, TNM: tumor node metastasis, NLR: neutrophil-to-lymphocyte ratio, Fib: fibrinogen, FAR: fibrinogen-to-albumin ratio, FPR: fibrinogen-to-prealbumin ratio. 
Figure 2. Pearson's correlation analyses. FPR was positively correlated with Fib $(r=0.673, p<0.001 ; A), F A R(r=0.622$, $p<0.001 ; B)$, and NLR $(r=0.206, p<0.001 ; C)$. FPR was negatively correlated with ALB $(r=-0.175, p=0.001 ; D)$ and PALB $(r=-0.713, p<0.001 ; E)$.

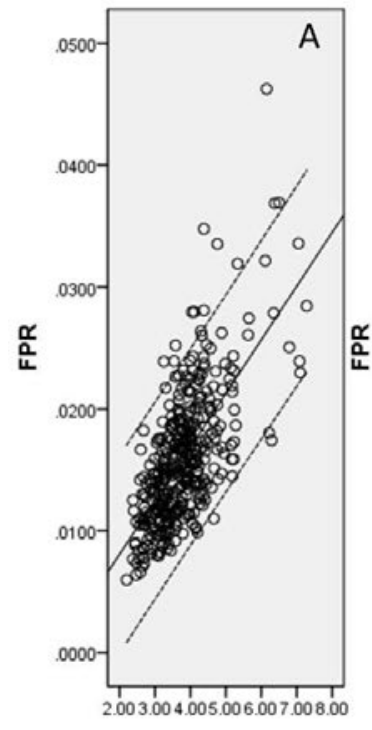

Fib

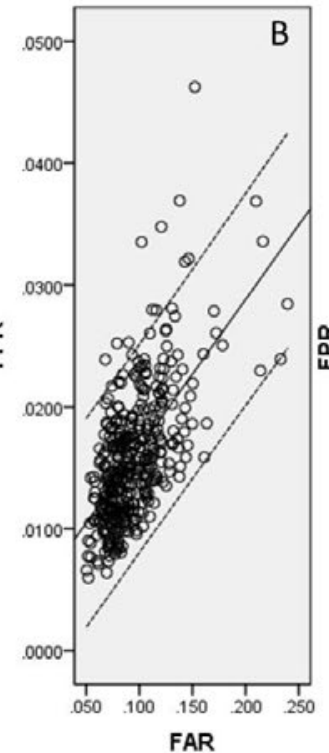

FAR

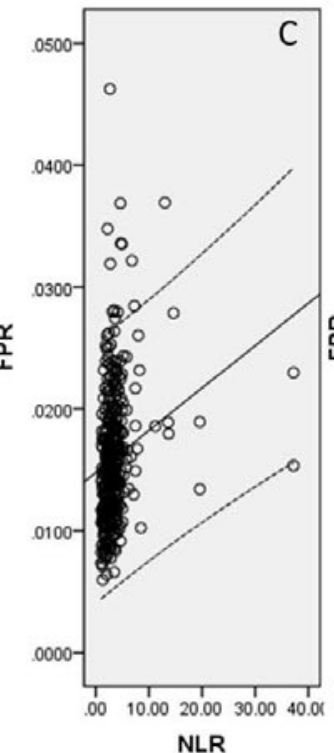

NLR
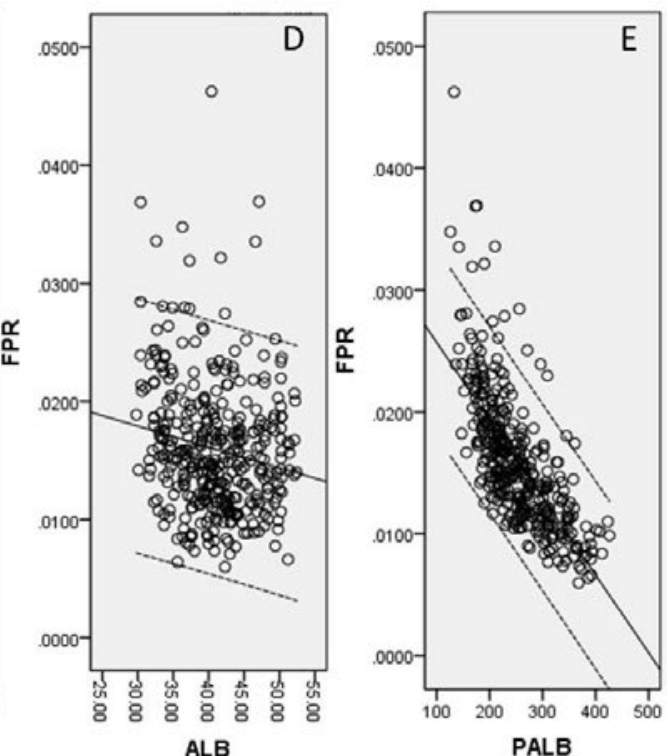

revealed that FPR (divided into three groups) was also significantly associated with CSS (Fig. 3I) and OS (Fig. 3J).

\section{Cox regression analysis}

Two models for Cox analyses (one for categorical variables and the other for continuous variables) were used. Both univariate analyses, using categorical and continuous variables, indicated that tumor length, vessel invasion, perineural invasion, TNM stage, NLR, Fib, ALB, PALB, FAR, and FPR were significantly associated with CSS and OS (Table S1). Multivariate Cox analyses (categorical and continuous) demonstrated that FPR was an independent prognostic factor in CSS (categorical: HR: 2.014, 95\% Cl: 1.504-2.697, p < 0.001 ; continuous per 0.01: HR: $1.438,95 \% \mathrm{Cl}$ : 1.154-1.793, $\mathrm{p}=0.001$ ) and OS (categorical: HR: $1.964,95 \% \mathrm{Cl}: 1.475-2.617, \mathrm{p}<0.001$; continuous per 0.01: HR: $1.429,95 \% \mathrm{Cl}: 1.146-1.781, \mathrm{p}=0.002$ ) (Table S2).

\section{ROC analysis}

The AUC areas were $0.739,0.711,0.712,0.653$, 0.608 , and 0.640 for FPR, FAR, Fib, NLR, ALB, and PALB, respectively (Fig. 1). Comparison of AUC areas regarding the FPR, FAR, Fib, NLR, ALB, and PALB in ESCC is shown in Table S3.

\section{Nomogram analysis}

A nomogram, using three independent prognostic factors (TNM, FAR, and FPR) in multivariate analyses, was used to predict the CSS and OS (Fig. 4). The 1-, 3-, and 5-year CSS and OS probability of ESCC patients could be predicted according to this nomogram model.

\section{DISCUSSION}

As far as we know, this is the first study in ESCC to indicate the prognostic value of FPR (Fib/PALB ratio). Our study revealed that pre-operative FPR serves as an independent prognostic factor for CSS (HR: 2.014, $95 \% \mathrm{Cl}: 1.504-2.697, \mathrm{P}<0.001)$ and OS (HR: 1.964 , $95 \% \mathrm{Cl}: 1.475-2.617, \mathrm{p}<0.001)$. Moreover, we first performed a nomogram using TNM, FAR, and FPR to predict the 1-, 3-, and 5-years CSS and OS probability in patients with ESCC.

The prognostic role of plasma Fib in EC is still controversial. Wakatsuki et al. $^{10}$ and Takeuchi et al. ${ }^{11}$ 
Figure 3. Kaplan-Meier CSS and OS curves. Kaplan-Meier curves regarding CSS ( $50.7 \%$ vs. 18.0\%, p $<0.001 ; A)$ and OS (48.0\% vs. $17.6 \%, p<0.001$; B) grouped by FPR. Kaplan-Meier curves regarding CSS (TNM I: $31.9 \%$ vs. $66.0 \%, p<0.001$, C; TNM II: $20.6 \%$ vs. $48.2 \%, p=0.001$, D; TNM III: $10.7 \%$ vs. $38.3 \%, p<0.001$, E) and OS (TNM I: $29.8 \%$ vs. $61.7 \%, p=0.001, F ;$ TNM II: $20.6 \%$ vs. $44.6 \%, p=0.002$, G; TNM III: $10.7 \%$ vs. $38.3 \%, p<0.001, \mathrm{H}$ ) in subgroup analyses based on TNM stage. KaplanMeier curves regarding CSS (69.2\% vs. $31.0 \%$ vs. $11.1 \%, p<0.001 ; \mathrm{l})$ and OS $(66.7 \%$ vs. $29.5 \%$ vs. $11.1 \%, p<0.001 ; \mathrm{J})$ grouped by FPR divided into three groups $(\leq 0.01,0.01-0.02$ and $>0.02)$.
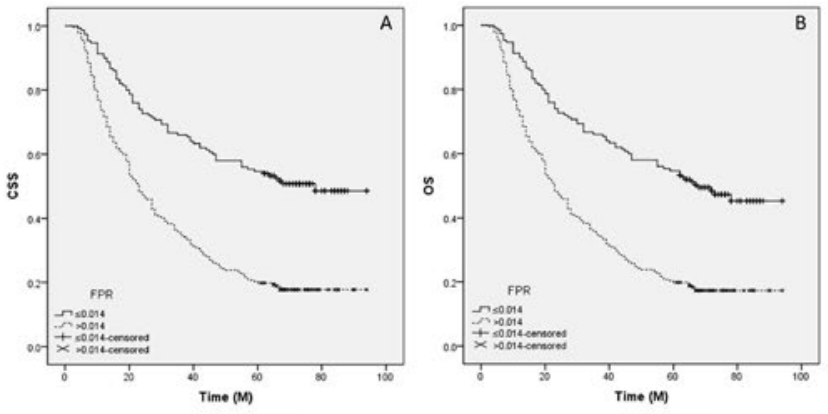

TNM III
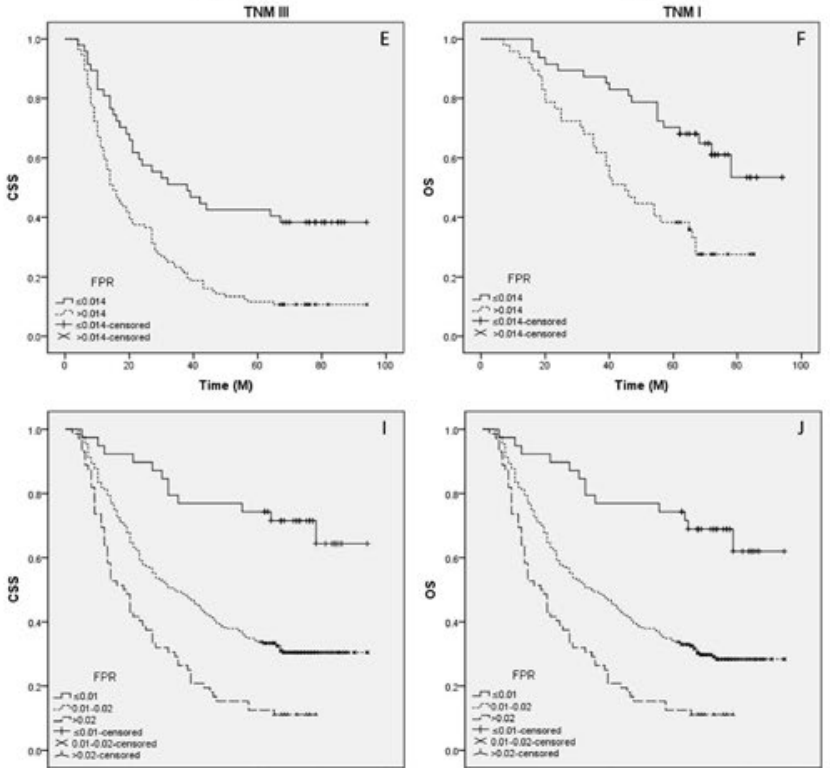

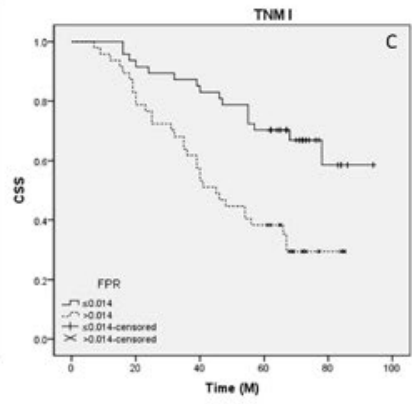

TNM II
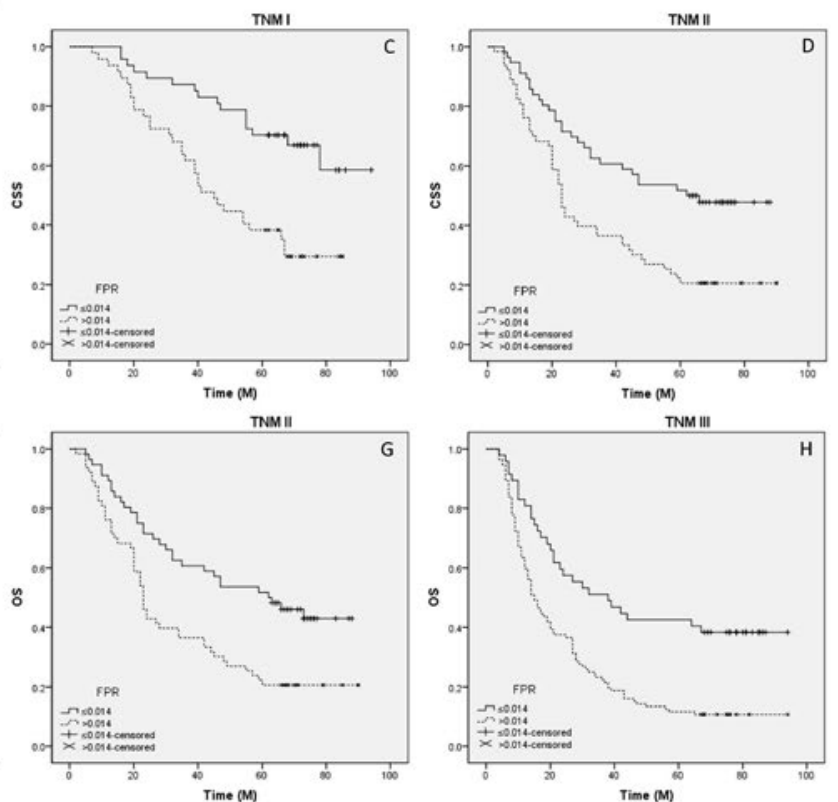

demonstrated that plasma Fib was a useful independent predictive indicator for prognosis in ESCC. Conversely, Li et al. ${ }^{12}$ revealed that Fib was not associated with the OS of ESCC. Recently, two meta-analyses revealed that plasma Fib was a prognostic indicator in patients with $\mathrm{EC}^{13,14}$. However, the present study revealed that Fib was not associated with CSS or OS in ESCC patients.

The prognostic value of $A L B$, as a nutritional indicator, has been confirmed in a variety of cancers, including patients with $\mathrm{EC}^{15,16}$. A new prognostic index in recent years was proposed with FAR for patients with $\mathrm{ESCC}^{17}$. PALB, as another important nutritional indicator, is more sensitive to malnutrition than ALB ${ }^{18,19}$. However, no study regarding the prognostic role of FPR has been assessed so far in ESCC patients. Moreover, the prognostic role in patients with ESCC between FPR (Fib/PALB) and FAR (Fib/ALB) is still unknown. In the current study, patients with lower levels of FPR ( $\leq$ 0.014 ) had better CSS ( $50.7 \%$ vs. $18.0 \%, p<0.001$ ) and OS ( $48.0 \%$ vs. $17.6 \%, p<0.001)$. FPR serves as an independent prognostic factor for CSS (HR: 2.014, 95\% Cl: 1.504-2.697, p < 0.001) and OS (HR: 1.964, $95 \% \mathrm{Cl}: 1.475-2.617, \mathrm{p}<0.001)$.

As we know, Fib, PALB, and ALB are all routinely tested blood indexes in daily clinical practice. In the present study, we have first explored the prognostic value of FPR comparing with Fib and FAR in ESCC. In our results, the AUC area was 0.739, 0.711, 0.712, 0.653, 0.608 , and 0.640 for FPR, FAR, Fib, NLR, ALB, and 
Figure 4. Nomogram analyses. A nomogram predicts 1-, 3-, and 5-year CSS (A) and OS (B) probability based on FAR, FPR, and TNM in patients with ESCC.

Points
TNM
FAR
FPR
Total Points
Linear Predictor
1-year CSS Probability
3-year CSS Probability
5-year CSS Probability

A

5-year CSS Probability

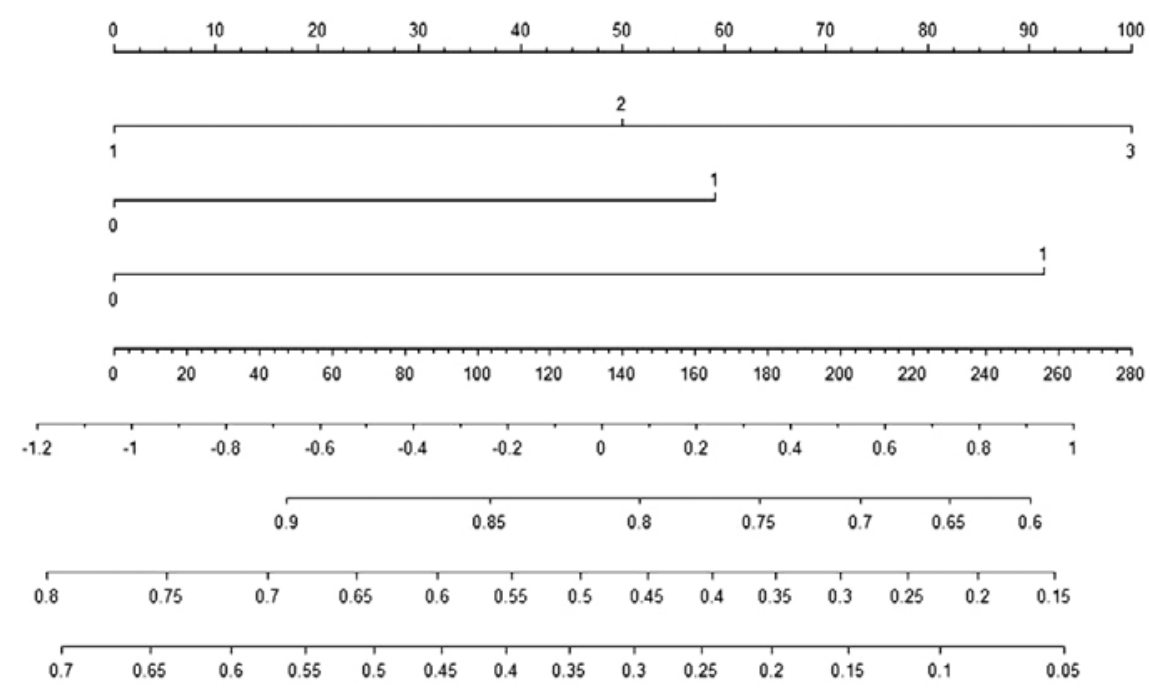

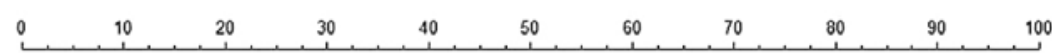

B

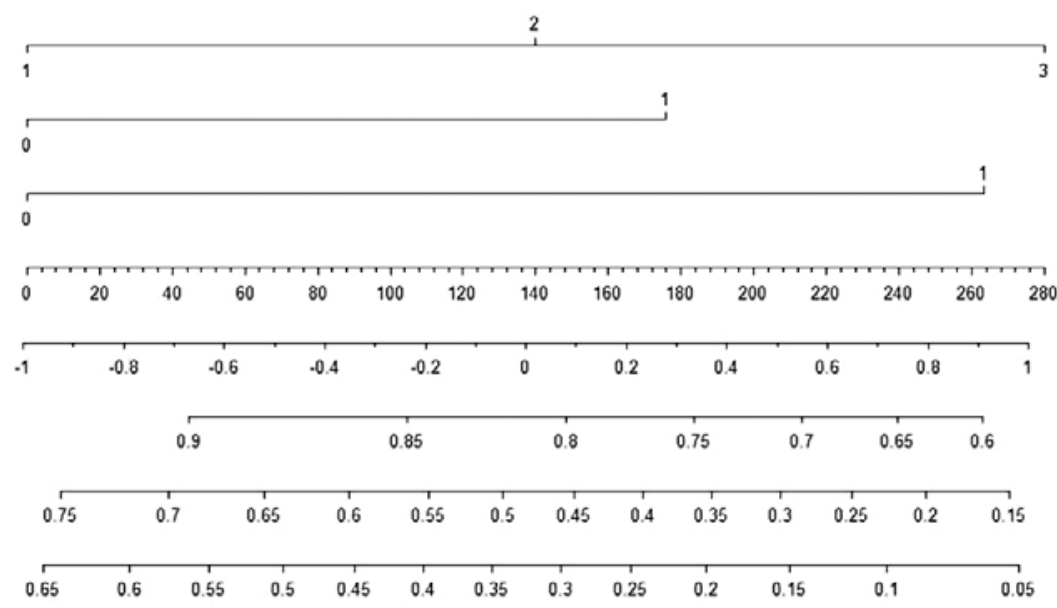

PALB, respectively. Moreover, patients with lower levels of FPR $(\leq 0.014)$ were associated with better CSS $(p<0.001)$.

Pre-operative neoadjuvant treatment (chemotherapy and/or radiotherapy) followed by surgery improves survival in several randomized control trials. The OEO2 trial in operable EC patients with pre-operative chemotherapy revealed that pre-operative chemotherapy followed by surgery prolonged the DFS (HR $=0.82,95 \%$ $\mathrm{Cl}: 0.71-0.95, \mathrm{p}=0.003)$ and $\mathrm{OS}(\mathrm{HR}=0.84,95 \% \mathrm{Cl}$ : $0.72-0.98, p=0.03)^{27}$. The CROSS trial including 366 patients with esophageal or junctional cancer also demonstrated that neoadjuvant chemoradiotherapy followed by surgery improved DFS (HR $=0.498,95 \%$ Cl: 0.357-0.693, $\mathrm{p}<0.001)$ and OS (HR $=0.657,95 \%$ $\mathrm{Cl}: 0.495-0.871, \mathrm{p}=0.003)^{28}$. However, the French trial with 195 EC patients in TNM Stages I and II (FFCD 9901) revealed that pre-operative chemoradiotherapy followed by surgery did not improve DFS ( $H R=0.92,95 \% \mathrm{Cl}: 0.66-1.30, \mathrm{p}=0.648)$ or OS (HR $=0.99,95 \% \mathrm{Cl}: 0.69-1.40, \mathrm{p}=0.94)^{29}$. The survival benefit of neoadjuvant chemoradiotherapy in patients with EC was confirmed in two meta-analyses ${ }^{30,31}$. In the current study, however, patients with any neoadjuvant treatment were excluded due to two reasons. 
On the one hand, pre-operative neoadjuvant therapy was controversial during that period (2006-2010). On the other hand, patients with pre-operative therapy might have a side effect on the levels of blood indexes.

There is no general consensus on post-operative adjuvant therapy in patients with EC. A randomized, controlled Phase III trial (JCOG9204) including 242 patients with ESCC revealed that surgery plus postoperative adjuvant chemotherapy showed better 5 -year DFS ( $55 \%$ vs. $45 \%, p=0.037$ ), but not for 5 -year OS (61\% vs. $52 \%, p=0.13)^{32}$. In another Japanese randomized trial (JCOG9907) for patients with Stage II/III ESCC, the results revealed that preoperative chemotherapy followed by surgery improved OS (HR $=0.973,95 \% \mathrm{Cl}: 0.54-0.99, \mathrm{p}=0.04)$ compared to post-operative adjuvant chemothera$\mathrm{py}^{33}$. In the current study, according to clinical criteria, a total of 115 patients (30.9\%) received post-operative adjuvant radiotherapy and/or chemotherapy. However, there were no statistical differences between patients with and without adjuvant treatment in CSS $(28.7 \%$ vs. $32.3 \%, p=0.129)$ or OS $(27.8 \%$ vs. $30.7 \%, p=0.152)$. The main reason was that patients with TNM I stage were included in the current study.

It is generally recognized that blood indexes including Fib and PALB may be influenced by various conditions. Therefore, the role of FPR would be more reliable. In the current study; moreover, we found positive correlations between FPR and NLR and negative correlations between FPR and ALB/PALB. Therefore, FPR is a combined indicator which can reflect a mixed prognostic role.

Some potential limitations of the current study should be mentioned. First, because this is a retrospective study in a single-center, potential selection bias cannot be excluded entirely. Second, patients with preoperative treatment were excluded from the study, which might have influenced the results for patients with ESCC 29,34 . Third, due to the lack of a prospective study design, the results of our study should be validated in more large-sample trials in the future. Any prognostic index must consider the AJCC necessary criteria for developing prognostic models. The AJCC committee proposed 13 inclusion and three exclusion criteria for a risk model ${ }^{35}$. However, we must acknowledge that our prognostic model was only developed, but not validated in this study. Therefore, the results of our study should be validated in future studies.

Our study indicated that FPR (cutoff point: 0.014) was an independent prognostic factor in patients with resectable ESCC. Patients with lower levels of FPR ( $\leq$ 0.014 ) had better CSS and OS than patients with higher levels of FPR ( $>0.014$ ).

\section{ACKNOWLEDGMENTS}

This study was funded by the Medical Health Science and Technology Project of the Zhejiang Provincial Health Commission (2018KY290, 2019RC129 and 2019RC128).

\section{SUPPLEMENTARY DATA}

Supplementary data are available at Revista de Investigación Clínica online (www.clinicalandtranslationalinvestigation.com). These data are provided by the corresponding author and published online for the benefit of the reader. The contents of supplementary data are the sole responsibility of the authors.

\section{REFERENCES}

1. Bray F, Ferlay J, Soerjomataram I, Siegel RL, Torre LA, Jemal A et al. Global cancer statistics 2018: GLOBOCAN estimates of incidence and mortality worldwide for 36 cancers in 185 countries. CA Cancer J Clin. 2018;68:394-424.

2. Siegel RL, Miller KD, Jemal A. Cancer statistics, 2019. CA Cancer J Clin. 2019;69:7-34.

3. Napier KJ, Scheerer M, Misra S. Esophageal cancer: a review of epidemiology, pathogenesis, staging workup and treatment modalities. World J Gastrointest Oncol. 2014;6:112-20.

4. Bedenne L, Michel P, Bouché O, Milan C, Mariette C, Conroy T, et al. Chemoradiation followed by surgery compared with chemoradiation alone in squamous cancer of the esophagus: FFCD 9102. J Clin Oncol. 2007;25:1160-8.

5. Domper Arnal MJ, Ferrández Arenas Á, Lanas Arbeloa Á. Esophageal cancer: risk factors, screening and endoscopic treatment in Western and Eastern countries. World J Gastroenterol. 2015; 21:7933-43.

6. Luo Y, Kim HS, Kim M, Lee M, Song YS. Elevated plasma fibrinogen levels and prognosis of epithelial ovarian cancer: a cohort study and meta-analysis. J Gynecol Oncol. 2017;28:e36.

7. Sun ZQ, Han XN, Wang HJ, Tang Y, Zhao ZL, Qu YL, et al. Prognostic significance of preoperative fibrinogen in patients with colon cancer. World J Gastroenterol. 2014;20:8583-91.

8. Ghanim B, Hoda MA, Klikovits T, Winter MP, Alimohammadi A, Grusch M, et al. Circulating fibrinogen is a prognostic and predictive biomarker in malignant pleural mesothelioma. $\mathrm{Br}$ J Cancer. 2014;110:984-90.

9. Zhao J, Zhao M, Jin B, Yu P, Hu X, Teng Y, et al. Tumor response and survival in patients with advanced non-small-cell lung can- 
cer: the predictive value of chemotherapy-induced changes in fibrinogen. BMC Cancer. 2012;12:330.

10. Wakatsuki K, Matsumoto S, Migita K, Ito M, Kunishige T, Nakade $\mathrm{H}$, et al. Preoperative plasma fibrinogen is associated with lymph node metastasis and predicts prognosis in resectable esophageal cancer. World J Surg. 2017;41:2068-77.

11. Takeuchi $H$, lkeuchi $S$, Kitagawa $Y$, Shimada A, Oishi $T$, Isobe $Y$ et al. Pretreatment plasma fibrinogen level correlates with tumor progression and metastasis in patients with squamous cell carcinoma of the esophagus. J Gastroenterol Hepatol. 2007; 22:2222-7

12. Li XH, Wang XP, Gu WS, Lin JH, Huang $H$, Kang $T$, et al. Clinical significance of preoperative thrombin time in patients with esophageal squamous cell carcinoma following surgical resection. PLoS One. 2015;10:e0140323.

13. Liu FT, Gao H, Wu CW, Zhu ZM. The association of plasma fibrinogen with clinicopathological features and prognosis in esophageal cancer patients. Oncotarget. 2017;8:93029-38

14. Lv GY, Yu Y, An L, Sun XD, Sun DW. Preoperative plasma fibrinogen is associated with poor prognosis in esophageal carcinoma: a meta-analysis. Clin Transl Oncol. 2018;20:853-61.

15. Miyata $H$, Yamasaki M, Kurokawa $Y$, Takiguchi S, Nakajima K, Fujiwara $Y$, et al. Prognostic value of an inflammation-based score in patients undergoing pre-operative chemotherapy followed by surgery for esophageal cancer. Exp Ther Med. 2011:2:879-85.

16. Kobayashi T, Oshima K, Yokobori T, Idetsu A, Hayashi Y, Hinohara RN, et al. Perioperative nutriture in esophageal cancer patients undergoing esophagectomy. Hepatogastroenterology. 2013;60:1311-6

17. Tan Z, Zhang M, Han Q, Wen J, Luo K, Lin P, et al. A novel blood tool of cancer prognosis in esophageal squamous cell carcinoma: the fibrinogen/albumin ratio. J Cancer 2017:8:1025-9.

18. Gonda K, Shibata M, Sato Y, Washio M, Takeshita H, Shigeta H, et al. Elevated neutrophil-to-lymphocyte ratio is associated with nutritional impairment, immune suppression, resistance to S-1 plus cisplatin, and poor prognosis in patients with stage IV gastric cancer. Mol Clin Oncol. 2017;7:1073-8.

19. Unal D, Orhan O, Eroglu C, Kaplan B. Prealbumin is a more sensitive marker than albumin to assess the nutritional status in patients undergoing radiotherapy for head and neck cancer. Contemp Oncol (Pozn). 2013;17:276-80

20. Yang YS, Shang OX, Yuan Y, Wu XY, Hu WP, Chen LQ et al. Comparison of long-term quality of life in patients with esophageal cancer after ivor-lewis, mckeown, or sweet esophagectomy. J Gastrointest Surg. 2019;23:225-31.

21. Raymond DP, Seder CW, Wright CD, Magee MJ, Kosinski AS, Cassivi SD, et al. Predictors of major morbidity or mortality after resection for esophageal cancer: a society of thoracic surgeons general thoracic surgery database risk adjustment model. Ann Thorac Surg. 2016;102:207-14

22. Kato $H$, Nakajima M. Treatments for esophageal cancer: a review. Gen Thorac Cardiovasc Surg. 2013;61:330-5

23. Rice TW, Rusch VW, Ishwaran H, Blackstone EH, Worldwide Esophageal Cancer Collaboration. Cancer of the esophagus and esophagogastric junction: data-driven staging for the seventh edition of the American joint committee on cancer/international union against cancer cancer staging manuals. Cancer. 2010;116:3763-73

24. Keteyian S], Patel M, Kraus WE, Brawner CA, McConnell TR, Piña $\mathrm{IL}$, et al. Variables measured during cardiopulmonary exercise testing as predictors of mortality in chronic systolic heart failure. J Am Coll Cardiol. 2016;67:780-9.

25. Mazumdar M, Smith A, Bacik J. Methods for categorizing a prognostic variable in a multivariable setting. Stat Med. 2003; 22:559-71.

26. Harrington D, D'Agostino RB Sr, Gatsonis C, Hogan JW, Hunter DJ, Normand ST, et al. New guidelines for statistical reporting in the journal. N Engl J Med. 2019;381:285-6

27. Allum WH, Stenning SP, Bancewicz J, Clark PI, Langley RE. Longterm results of a randomized trial of surgery with or without preoperative chemotherapy in esophageal cancer. J Clin Oncol. 2009;27:5062-7.

28. van Hagen $P$, Hulshof MC, van Lanschot J], Steyerberg EW, van Berge Henegouwen MI, Wijnhoven BP, et al. Preoperative chemoradiotherapy for esophageal or junctional cancer. N Engl J Med. 2012:366:2074-84.

29. Mariette C, Dahan L, Mornex F, Maillard E, Thomas PA, Meunier $B$, et al. Surgery alone versus chemoradiotherapy followed by surgery for stage I and II esophageal cancer: final analysis of randomized controlled phase III trial FFCD 9901. J Clin Oncol. 2014;32:2416-22.

30. Sjoquist KM, Burmeister BH, Smithers BM, Zalcberg JR, Simes RJ, Barbour A, et al. Survival after neoadjuvant chemotherapy or chemoradiotherapy for resectable oesophageal carcinoma: an updated meta-analysis. Lancet Oncol. 2011;12:681-92.

31. Lv J, Cao XF, Zhu B, Ji L, Tao L, Wang DD, et al. Effect of neoadjuvant chemoradiotherapy on prognosis and surgery for esophageal carcinoma. World J Gastroenterol. 2009; 15:4962-8.

32. Ando $N$, lizuka $T$, Ide $H$, Ishida $K$, Shinoda $M$, Nishimaki $T$, et al. Surgery plus chemotherapy compared with surgery alone for localized squamous cell carcinoma of the thoracic esophagus: a Japan clinical oncology group study - JCOG9204. J Clin Oncol. 2003:21:4592-6.

33. Ando N, Kato H, Igaki H, Shinoda M, Ozawa S, Shimizu H, et al A randomized trial comparing postoperative adjuvant chemotherapy with cisplatin and 5-fluorouracil versus preoperative chemotherapy for localized advanced squamous cell carcinoma of the thoracic esophagus (JCOG9907). Ann Surg Oncol. 2012; 19:68-74

34. Shapiro J, van Lanschot JJ, Hulshof MC, van Hagen $P$, van Berge Henegouwen MI, Wijnhoven BP, et al. Neoadjuvant chemoradiotherapy plus surgery versus surgery alone for oesophageal or junctional cancer (CROSS): long-term results of a randomised controlled trial. Lancet Oncol. 2015:16:1090-8.

35. Kattan MW, Hess KR, Amin MB, Lu Y, Moons KG, Gershenwald $\mathrm{JE}$, et al. American joint committee on cancer acceptance criteria for inclusion of risk models for individualized prognosis in the practice of precision medicine. CA Cancer J Clin. 2016; 66:370-4. 